\title{
ALLOPURINOL-INDUCED STEVENS-JOHNSON SYNDROME AND TOXIC EPIDERMAL NECROLYSIS: A CASE REPORT
}

\author{
MANIK CHHABRA*, ANKIT GAUR \\ Department of Pharmacy Practice, Indo-Soviet Friendship College of Pharmacy, Moga, Punjab, India. Email: manikchhabra57@gmail.com
}

Received: 01 September 2018, Revised and Accepted: 02 November 2018

\begin{abstract}
Allopurinol is used for the treatment of gout and related conditions; it is associated with various adverse drug reactions (ADRs) such as StevensJohnson syndrome (SJS) and toxic epidermal necrolysis (TEN). A 50-year-old female was presented to the emergency ward with chief complaints of reddish pinpoint lesions over the back, chest, abdomen, and lower limb-upper limb for the past 6 days. She was diagnosed with SJS. On medication interview, it was revealed that she was on allopurinol therapy, which she took 5 days back as a self-medication. The World Health Organization - Uppsala Monitoring Centre (WHO) scale was used to access the causality assessment, ADR was found to be probable. The drug was withdrawn from the therapeutic regimen of the patient. The patient was discharged after 25 days from the hospital. Allopurinol has the strongest association with SJS with TEN. There should be a screening of HLA-B 5801 antigens before commencing the allopurinol therapy to the patients.
\end{abstract}

Keywords: Allopurinol, Stevens-Johnson Syndrome, Toxic epidermal necrolysis, Corticosteroid therapy, Mucocutaneous adverse drug reactions.

(C) 2019 The Authors. Published by Innovare Academic Sciences Pvt Ltd. This is an open access article under the CC BY license (http://creativecommons. org/licenses/by/4. 0/) DOI: http://dx.doi.org/10.22159/ajpcr.2019.v12i2.29448

\section{INTRODUCTION}

Whenever a drug therapy is initiated, risk is taken beside the therapeutic effects and that risk may result in adverse drug reactions (ADRs). ADRs are a sixth leading cause of death worldwide [1]. Risk of ADR with marketed pharmaceutical formulation is assessed through voluntary reporting of ADR by various health-care professionals who further decides the marketing of that particular pharmaceutical formulation [2]. The most serious ADRs are the mucocutaneous ADRs through their rate of occurrence is relatively low but are potentially fatal and endanger patient's life [3]. Most common culprits of mucocutaneous ADR are antiepileptic drugs, antimicrobial drugs, sulfa drugs, nonsteroidal antiinflammatory drugs (NSAIDs), and antirheumatic drugs [4]. StevensJohnson syndrome (SJS) and toxic epidermal necrosis (TEN) are categorized under cutaneous ADRs. SJS and TEN are rare, responsible for around 1 or 2/100,000 cases annually and are potentially fatal due to which they are considered as a medical emergency. In the year 1922 , SJS was described for the $1^{\text {st }}$ time in two young boys [5]. As per the criteria laid by international group of dermatologist, SJS is an acute mucocutaneous adverse reaction, characterized by erosion or bullae with erythematous base. Reaction covering $\geq 20 \%$ of body surface area with three distinct involvement of anatomical areas, peeling of area of skin larger than $3 \mathrm{~cm}$, frequent involvement of mucous membrane with character of appearance of tenderness within $48 \mathrm{~h}$ of onset of rash, drug-induced basal necrosis TEN, and specimen compatible with SJS [6]. This definition excludes staphylococcal skin syndrome and appearance of the lesion that has been exposed to sunlight. Both SJS and TEN relatively affect 1 or $2 / 1000,000$ people every year [7]. Habitually patients diagnosed with SJS-TEN complain of burning rash with symmetric origin on the face and upper part of the torso. The rash starts to begin as macules and on progression, it develops into papules, vesicles bullae, and urticarial plaques. Later on, signs of mucosal involvement include erythema, edema, sloughing, blistering, ulceration, and necrosis [8].

\section{CASE REPORT}

A 50-year-old female presented to the emergency department with reddish pinpoint lesions over the back, chest, abdomen, and lower limb-upper limb for the past 6 days was slowly progressing and had a complaint of gradually increasing oral ulceration for the past 5 days. History dates back to 5 to 6 days back, she took allopurinol for joint pain as a self-medication after 2 days of administration of allopurinol, she developed erythematous maculopapular rashes all over the body which started over upper limbs and gradually involved chest, abdomen, back, and lower limb gradually, she developed ulceration over mouth and followed by blistering of the lips. She also developed redness of eye along with photophobia and mucopurulent discharge from both eyes. Nikolsky's sign appeared to be positive. The patient had a history of epistaxis and burning micturition; the patient case of hypothyroidism previously and was on irregular medication for the past 3 months. On examination, the patient was semiconscious and her blood pressure was 140/90 $\mathrm{mmHg}$, respiratory rate was 18 per min, pulse rate was 78 beats per minute, and oral temperature was $97.8^{\circ} \mathrm{F}$ on day admission, but after 3 days, she turned febrile with high-grade fever, her oral temperature was $103^{\circ} \mathrm{F}$ which was relieved by administration of paracetamol. All the laboratories investigations were almost normal, her hemoglobin, mean corpuscular volume, mean corpuscular hemoglobin, and hematocrit were low-indicating megaloblastic anemia, urine examination was also normal, IgE and IgM antibodies were negative, random blood sugar was $165 \mathrm{mg} / \mathrm{dL}$, RFT were quite normal, and total serum protein and albumin were normal. Her liver enzymes were elevated, serum glutamic oxaloacetic transaminase was found to be $75 \mathrm{IU} / \mathrm{L}$, SGPT was $84 \mathrm{IU} / \mathrm{L}$, and $25 \%$ of body surface was affected. Differential diagnosis like herpes simplex virus autoimmune disorders of skin like pemphigus vulgaris, fix drug eruptions, erythema multiforme were ruled out. The final diagnosis of SJS was made. The causality of ADR and allopurinol was accessed using the WHO causality scale and ADR was found to be probable. Details of the drug therapy administered to the patient are shown in Table 1.

The patient's condition was improved and was discharged after 25 days from the hospital. The patient was advised not to administer allopurinol in the future. Prediction of course of a disease was done with score TEN scale to predict the percentage of mortality rate with the risk factor associated with SJS-TEN. Score TEN is well described in Tables 2 and 3 [9]. 
Table 1: Drug therapy administered to the patient

Inj. pheniramine $22.75 \mathrm{mg}$ was given IV twice a day Inj. hydrocortisone $100 \mathrm{mg}$ IV was given 3 times a day Mouthwash hexidine was given in diluted form 3 times a day GV (GV dye) paint 1\% was applied over erosions

Tab. fluconazole $100 \mathrm{mg}$ was given once a day

Paraffin oil was applied over the body after cleaning Eye ointment of carboxyl methyl cellulose $0.5 \%$ was applied 3 times daily to both eyes

Tab. paracetamol $650 \mathrm{mg}$ was given as SOS

Tab. linezolid $600 \mathrm{mg}$ was given 2 times a day

Calamine lotion was applied during night time over the whole body

GV: Gentian violet

Table 2: The score TEN scale used for calculations of the risk factor

\begin{tabular}{lll}
\hline Risk factors & $\mathbf{0}$ & $\mathbf{1}$ \\
\hline Age & $<40$ years & $>40$ years \\
Associated malignancy & No & Yes \\
Heart rate (beats/min) & $<120$ & $>120$ \\
Serum BUN (mg/dL) & $<28$ & $>28$ \\
Compromised BSA & $<10 \%$ & $>10 \%$ \\
Serum bicarbonate mEq/L & $>20$ & $<20$ \\
Serum glucose & $<252$ & $>252$ \\
\hline
\end{tabular}

Table 3: Mortality rate with number of risk factor calculated by score TEN scale

\begin{tabular}{ll}
\hline Number of risk factors & Mortality rate (\%) \\
\hline $0-1$ & 3.2 \\
2 & 12.1 \\
3 & 35.3 \\
4 & 58.3 \\
5 or more & $>90$ \\
\hline
\end{tabular}

\section{DISCUSSION}

Long back in the year 1922, SJS was described for the $1^{\text {st }}$ time as rare, unspecified epidermal eruptions characterized by pyrexia, swelling of buccal mucosa, and austere conjunctivitis by American pediatricians Albert Mason Stevens and Frank Chambliss Johnson in two young boys of age 7 and 8 years. Both these cases were misdiagnosed by primary physician as hemorrhagic measles [10]. The incidence of SJS is relatively very low. Multiple etiologies are involved in the pathophysiology of this syndrome. Various drugs such as NSAIDs, sulfonamides, and antiepileptic, including allopurinol, several infectious agents, and idiopathic reasons are responsible for the cause of SJS [11]. Their strong evidence of the association between HLA-B 5801 and occurrence of SJSTEN allopurinol induced [12]. The mortality rates are higher in younger and older age. There are various studies, suggesting that patients suffering from HIV are at higher risk of developing SJS and TEN. The terminology of SIS and TEN is assigned as if the $10 \%$ of the body surface is covered than the subject is having SJS similarly if $10-30 \%$ of body surface area than SJS and TEN overlaps and $>30 \%$ is said to be TEN [13].

An approach to treatment is based on the symptoms and is supportive begins with cessation of the culprit drug responsible for its etiology [14]. Treatment was initiated with the management of airways, fluid electrolyte management, and wound care. For skin, lesions, or skin loss, patients are treated as burn patients with extra care and are shifted to burn care units. Paradesi et al. found that patients who were on etanercept therapy were more promising with the median healing time of 8.5 days [15]. There are studies, which are not favoring the use of corticosteroids, but still, these are used in the management of SJS TEN.

\section{CONCLUSION}

Allopurinol has the strongest association with SJS-TEN. Early diagnosis of SJS-TEN and initiation of therapy with the systemic corticosteroid and supportive medication proved to be useful in the management of the patients. An early approach is to withdraw the drug and initiation of supportive measures [16-21]. Better how there should be a screening of HLA-B 5801 before commencing the therapy of allopurinol to the patients.

\section{ACKNOWLEDGMENT}

The authors acknowledge late Dr. Kanad Deepak PharmD for his continuous motivation toward research and Department of Pharmacy Practice, ISF College of Pharmacy, Moga, Punjab, India.

\section{ETHICAL DECLARATION}

Authors informed consent was taken from the patient before data collection stating that no identity of the patient will be disclosed.

\section{AUTHORS' CONTRIBUTIONS}

Manik Chhabra did acquisition of data, analysis of case report, and drafting of case report and Ankit Gaur did critical revision of manuscript.

\section{CONFLICTS OF INTEREST}

Authors declare that they have no conflicts of interest.

\section{REFERENCES}

1. Lazarou J, Pomeranz BH, Corey PN. Incidence of adverse drug reactions in hospitalized patients: A meta-analysis of prospective studies. JAMA 1998;279:1200-5

2. World Health Organization. The Importance of PharmacovigilanceSafety Monitoring of Medicinal Products. Who; 2002. Available from: http://www.apps.who.int/medicinedocs/pdf/s4893e/s4893e.pdf.

3. Srinivasan B, Agarwal S, Iyer G. Stevens-Johnson syndrome, toxic epidermal necrolysis and other mucocutaneous syndromes. In: Emergencies of the Orbit and Adnexa. Vol. 4. New Delhi, India: Springer; 2017. p. 217-24.

4. Gomes ER, Kuyucu S. Epidemiology and risk factors in drug hypersensitivity reactions. Curr Treat Options Allergy 2017;4:239-57.

5. Harr T, French LE. Toxic epidermal necrolysis and stevens-johnson syndrome. Orphanet J Rare Dis 2010;5:39.

6. Li LF, Ma C. Epidemiological study of severe cutaneous adverse drug reactions in a city district of China. Clin Exp Dermatol 2006;31:642-7.

7. Frey N, Jossi J, Bodmer M, Bircher A, Jick SS, Meier CR, et al. The epidemiology of stevens-johnson syndrome and toxic epidermal necrolysis in the UK. J Invest Dermatol 2017;137:1240-7.

8. Mockenhaupt M, Messenheimer J, Tennis P, Schlingmann J. Risk of stevens-johnson syndrome and toxic epidermal necrolysis in new users of antiepileptics. Neurology 2005;64:1134-8.

9. Sorrell J, Anthony L, Rademaker A, Belknap SM, Callahan S, West DP, et al. Score of toxic epidermal necrosis predicts the outcomes of pediatric epidermal necrolysis. Pediatr Dermatol 2017;34:433-7.

10. Bohigian GM. The history of stevens-johnson syndrome. Hist Ophthal Intern 2015;1:87-9. 
11. Guillaume JC, Roujeau JC, Revuz J, Penso D, Touraine R. The culprit drugs in 87 cases of toxic epidermal necrolysis (Lyell's syndrome). Arch Dermatol 1987;123:1166-70.

12. Hung SI, Chung WH, Liou LB, Chu CC, Lin M, Huang HP, et al. HLA-B*5801 allele as a genetic marker for severe cutaneous adverse reactions caused by allopurinol. Proc Natl Acad Sci U S A 2005;102:4134-9.

13. Schneider JA, Cohen PR. Stevens-johnson syndrome and toxic epidermal necrolysis: A concise review with a comprehensive summary of therapeutic interventions emphasizing supportive measures. Adv Ther 2017;34:1235-44.

14. Sukasem C, Katsila T, Tempark T, Patrinos GP, Chantratita W. Drug-induced stevens-johnson syndrome and toxic epidermal necrolysis call for optimum patient stratification and theranostics via pharmacogenomics. Annu Rev Genomics Hum Genet 2018;19:329-53.

15. Paradisi A, Abeni D, Bergamo F, Ricci F, Didona D, Didona B, et al. Etanercept therapy for toxic epidermal necrolysis. J Am Acad Dermatol 2014;71:278-83

16. Tripathi RK, Chhabra M, Rathore MS. Cost variations among gastric acid lowering medications used for treatment of gastroesophageal reflux disease (GERD). Asian J Pharm Clin Res 2018;4:452-5.

17. Jangra MS, Chhabra M. Cycloserine induced suicidal tendencies and kanamycin induced ototoxicity in Indian MDR-TB patient: A case report. Curr Drug Saf 2018;13:211-3.

18. Chhabra M, Gaur A. A case report on acute copper sulfate poisoning in tertiary teaching care hospital. World J Pharm 2018;7:1472-76.

19. Sharma A, Gupta TK, Rathore MS, Chhabra M, Gaur A. Drug utilization study on oral hypertensive medication patients and assessment of medication adherence to JNC-8 guidelines in North Indian tertiary care hospital: A cross-sectional study. Res Rev J Hosp Clin Pharm 2017;3:5-12.

20. Jena SS, Jena M, Patro N, Mishra S, Panda M, Dash M. Patterns of prescription and ADR monitoring of drugs in the management of neuropathic pain in a tertiary care teaching hospital. Int J Pharm Pharm Sci 2014;6:246-51

21. Raut AS, Pawar AT, Pankaj MA, Srivastava PR, Mishra AD. Clinical pattern and severity of cutaneous adverse drug reactions. Int J Pharm Pharm Sci 2013;5:612-6. 